\title{
Oncoplastic Partial \\ Mastectomy/Lumpectomy with Oncoplastic Closure
}

National Cancer Institute

\section{Source}

National Cancer Institute. Oncoplastic Partial Mastectomy/Lumpectomy with Oncoplastic

Closure. NCl Thesaurus. Code C107212.

A surgical procedure in which there is full-thickness excision of the malignant breast tumor and surrounding breast parenchyma to allow resection with wide surgical margins, while the remaining fibroglandular breast tissue is transposed to preserve the breast shape and cosmetic appearance in the best possible way. 